\title{
Dolichoectatic Basilar Artery Treated by Reducing Hemodynamic Stress
}

\author{
-Report of Two Cases-
}

\author{
Katsumi Matsumoto, Kazuo Yamada, Toru HayaKawa, \\ Kazuo KATAOKA, Kazumi YAMAMOTO, Takanori ONISHI, \\ Mamoru ITO and Heitaro MOGAMI
}

Department of Neurosurgery, Osaka University Medical School, Osaka

\begin{abstract}
Two cases of dolichoectatic basilar artery with a mass effect to the brainstem structure were treated by reducing hemodynamic stress. In one case, angiograms showed the dolichoectatic basilar artery creating a turbulent flow in the vertebrobasilar junction, and unilateral vertebral artery clipping in addition to posterior fossa decompression was performed. The other case, a combination with bilateral internal carotid artery occlusion, underwent bilateral superficial temporal artery-middle cerebral artery anastomoses. The possible surgical treatments of dolichoectasia are discussed.
\end{abstract}

Key words: dolichoectasis, basilar artery, hemodynamic stress

\section{Introduction}

Dolichoectasia of the basilar artery has often been found to be a mass lesion, with clinical manifestation including progressive cerebellar signs, brainstem dysfunction, and cranial nerve palsies. ${ }^{1-4,7,9,10,12,14,15)}$ Brainstem atrophy has also been observed in autopsy cases. ${ }^{12)}$ Direct surgery to this lesion is difficult, so there are few reports of surgical treatment. We report here two cases of dolichoectatic basilar artery treated by indirect surgery to reduce hemodynamic stress on the lesion.

\section{Case Reports}

Case 1: A 46-year-old male, with a 2-year history of hypertension, developed walking problems and suffered dizziness 1 year prior to admission. Ten months later, he noted numbness of the right hand and difficulty in swallowing. Neurological examination on admission revealed numbness of the thumb, a decrease in the left gag reflex, and positive Romberg sign. A computed tomographic (CT) scan showed a strongly enhanced mass lesion in the right lateral aspect of the pons and widening of the prepontine cistern (Fig. 1). A four-vessel study was performed, in which bilateral vertebral angiograms showed an enlarged, tortuous basilar artery, relatively small right vertebral artery, and a turbulent flow in the dilated vertebrobasilar junction caused by blood supply from the right vertebral artery (Fig. 2A). No abnormalities were seen in the carotid arteries. The turbulent flow was also observed by transcranial Doppler sonography. Hypertension and turbulent flow in the vertebrobasilar junction were thought to aggravate the dolichoectasia.

On May 24, 1988, a wide suboccipital craniectomy for posterior fossa decompression, and clipping of the right vertebral artery at the part distal to the right posterior inferior cerebellar artery to reduce the tur-

Received November 9, 1989; Accepted April 12, 1990

Authors' present addresses: K. Matsumoto, M.D., Max-Planck Institute für Neurologische Forschung, Köln, Germany; K. Kataoka, M.D., Department of Neurosurgery, Kinki University School of Medicine, Sayama, Osaka, Japan; K. Yamamoto, M.D., Department of Neurosurgery, Ohno Memorial Hospital, Osaka, Japan; M. Ito, M.D., Department of Neurosurgery, Teramoto Memorial Hospital, Kawachinagano, Osaka, Japan. 


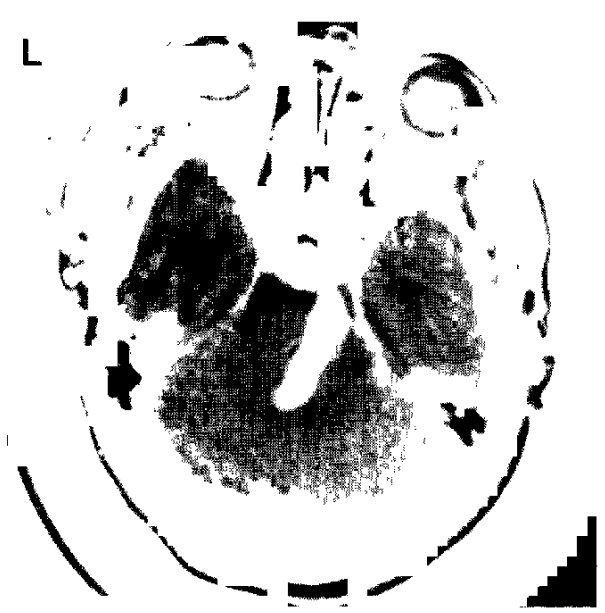

Fig. 1 Case 1. Preoperative CT scan, showing a club-shaped, strongly enhanced mass lesion in the right lateral aspect of the pons, and widening of the prepontine cistern.

bulent flow were performed. During the operation, a secondary arachnoid cyst was recognized and part of the wall opened. The postoperative course was uneventful. Six months after the operation, the gait and swallowing disturbance, dizziness, and numbness of the right hand were alleviated, although the hypertension was unaffected. A left vertebral angiogram revealed the reduced caliber of the basilar artery (Fig. 2B).

Case 2: Six years prior to admission, a 14-year-old boy suffered from right hemiparesis and was diagnosed as having bilateral carotid artery occlusion at the neck segment. The bilateral middle cerebral arteries (MCAs) and anterior cerebral arteries were filled through the posterior communicating arteries, and the basilar artery showed a slightly enlarged caliber but not fusiform dilatation.

A left superficial temporal artery (STA)-MCA anastomosis was performed on October 21, 1980, and the postoperative course was satisfactory until September, 1986, when he suffered floating sensation and generalized convulsion. A CT scan showed a strongly enhanced mass lesion in the prepontine and ambient cisterns (Fig. 3). Left vertebral angiograms revealed a dolichoectatic basilar artery, mainly fed by the left vertebral artery. The patent left STAMCA anastomosis supplied a good flow to the left hemisphere, and the blood flow in the right hemisphere was supplied from the basilar artery via the posterior communicating artery (Fig. 4A).

In October, 1986, a right STA-MCA anastomosis was performed to help the collateral blood supply from the posterior to anterior circulation and subsequently to reduce the blood flow in the basilar artery. He did well postoperatively, and intra-arterial digital subtraction angiograms taken 10 months after the operation revealed patency of the bilateral STAMCA anastomoses and a reduction in the size of the dolichoectasia of the basilar artery (Fig. 4B).

\section{Discussion}

Tortuous elongation and distension of the intracranial arteries is a rare condition, first named dolichoectasia by Sacks and Lindenberg. ${ }^{12)}$ Other
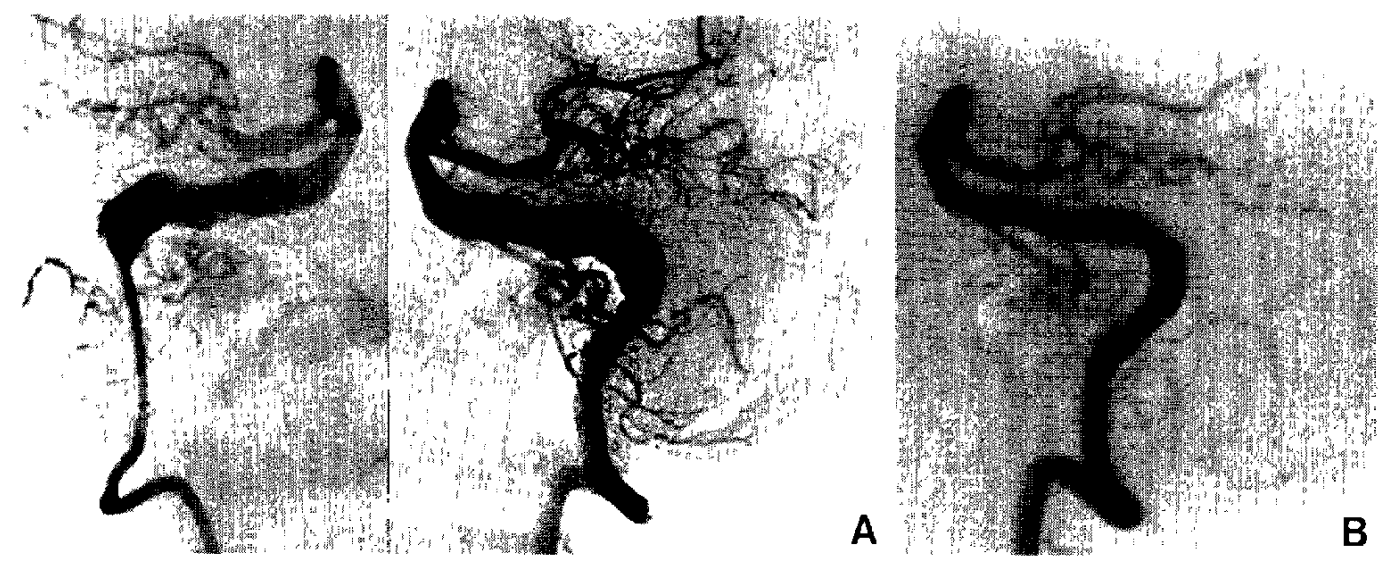

Fig. 2 Case 1. A: Preoperative right (left) and left (right) vertebral angiograms, lateral views, showing an elongated, dilated basilar artery, and the right vertebral artery being smaller than the left. Inhomogenous staining of the basilar artery due to the turbulent flow can also be observed on the right lateral view. B: Left vertebral angiogram taken 6 months after the operation, lateral view, revealing the reduced caliber of the basilar artery. 


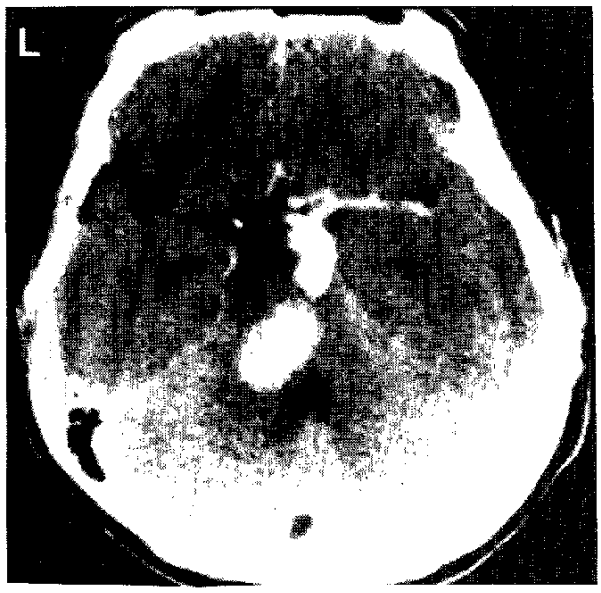

Fig. 3 Case 2. CT scan, showing a club-shaped, strongly enhanced mass lesion in the prepontine and ambient cisterns.

terms, such as megadolichobasilar anomaly, ${ }^{1,3,4)}$ or arteriectasis, ${ }^{7}$ have also been used. The lesion occurs as a result of atherosclerosis, ${ }^{15}$ however, dolichoectasia may not evolve with atherosclerosis. ${ }^{12)}$ It also occurs in association with moyamoya disease, ${ }^{14)}$ EhlersDanlos syndrome, ${ }^{2)}$ and EEC syndrome (ectrodactyly, ectodermal dysplasia, and clefting of the lip or palate) ${ }^{10)}$ Clinically, dolichoectasia mainly manifests a mass effect to the cranial nerves and brainstem structures, ${ }^{1.3,4,7,7,9,10,12.14,15)}$ and rarely causes subarachnoid hemorrhage. Because it involves vessels over a long distance and many important perforating arteries to the brainstem, direct surgical treatment, such as clipping and trapping, is difficult.

Previous reports have indicated that hemody- namic factors play an important role in the development of the intracranial dolichoectatic artery. Hypertension is often associated with dolichoectasia. ${ }^{7,12)}$ In one case of moyamoya disease, internal carotid artery dolichoectasia was associated with bilateral MCA stenoses. ${ }^{14)}$ There are many reports of saccular cerebral aneurysm related to hemodynamics..$^{5,6,8,11,13)}$ Considering the infrequency of subarachnoid hemorrhage, in contrast to saccular aneurysm, indirect surgery to alleviate the hemodynamic stress may be effective for this disease. In Case 1, two hemodynamic factors, the turbulent flow in the vertebrobasilar junction and essential hypertension, were considered to exert hemodynamic stress on the dolichoectasia. Proximal ligation of the right vertebral artery resulted in reduction of turbulent flow, although microemboli formation due to turbulent flow might be a cause of yertebrobasilar insufficiency. In Case 2, a right STA-MCA anastomosis was effective in diminishing the blood flow and the caliber of the basilar artery.

In conclusion, hemodynamic factors may play an important role in the development of dolichoectasia, although the pathogenesis of the lesion varies. The reduction of hemodynamic stress is considered to be effective, as in our cases.

\section{References}

1) Azar-Kia B, Palacios E, Spak M: The megadolichobasilar artery anomaly and expansion of the internal auditory meatus. Neuroradiology 11: 109-111, 1976

2) Bannerman RM, Grat CJ, Upson JF: Ehlers-Danlos syndrome. Brit Med J 3: 558-559, 1967

3) Boeri R, Passerini A: The megadolichobasilar anoma-
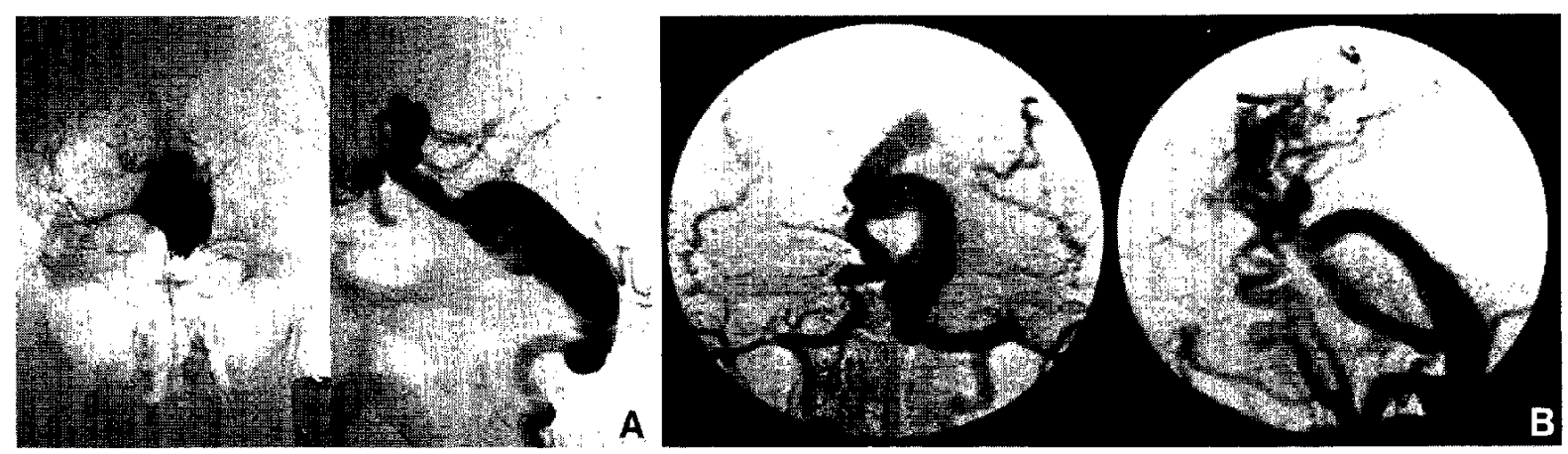

Fig. 4 Case 2. A: Left vertebral angiograms, anteroposterior (left) and lateral (right) views, showing the dolichoectatic basilar artery, and the right MCA filled through the posterior communicating artery. B: Intra-arterial digital subtraction angiograms taken 10 months after right STA-MCA anastomosis, anteroposterior (left) and lateral ( $r i g h t$ ) views, revealing a reduction in diameter of the dolichoectatic basilar artery and full patency of the anastomosis. 
ly. J Neurol Sci 1: 475-484, 1964

4) Frasson F, Ferrari G, Fugazzola C, Fiaschi A: Megadolichobasilar anomaly causing brainstem syndrome. A case report. Neuroradiology 13: 279-281, 1977

5) Hashimoto N, Handa H, Nagata I, Hazama F: Experimentally induced cerebral aneurysms in rats: Part $V$. Relation of hemodynamics in the circle of Willis to formation of aneurysms. Surg Neurol 13: 41-45, 1980

6) Hayashi S, Arimoto T, Itakura T, Fujii T, Nishiguchi $\mathrm{T}$, Komai $\mathrm{N}$ : The association of intracranial aneurysms and arteriovenous malformation of the brain. Case report. J Neurosurg 55: 971-975, 1981

7) Hulten-Gyllensten IL, Lof stedt S, von Reis G: Observations on generalized arteriectasis. Acta Med Scand 163: $125-131,1959$

8) Kodama N, Suzuki J: Moyamoya disease associated with aneurysm. $J$ Neurosurg 48: 565-569, 1978

9) Lye RH: Ectatic basilar artery: An unusual cause of trigeminal neuralgia, in Sami M (ed): Surgery in and around the Brain Stem and the Third Ventricle. Berlin, Springer, 1986, pp 280-284

10) Miner ME, Rea GL, Handel S, Bertz J: Trigeminal neuralgia due to dolichoectasia: Angiographic and CT findings in a patient with EEC syndrome. Neuroradiology 20: 163-166, 1980
11) Nukui $H$, Nagaya $T$, Miyagi $O$, Tamada $J$, Kaneko M, Sasaki H, Mitsuka S, Kawafuchi J, Kohno N, Kanoh T: Development of new aneurysm and enlargement of small aneurysm. Report of seven cases. Neurol Med Chir (Tokyo) 22: 437-445, 1982 (in Japanese)

12) Sacks JG, Lindenberg R: Dolicho-ectatic intracranial arteries: Symptomatology and pathogenesis of arterial elongation and distention. Johns Hopkins Med $J$ 125: 95-106, 1969

13) Tognetti F, Limoni P, Testa C: Aneurysm growth and hemodynamic stress. Surg Neurol 20: 74-78, 1983

14) Yamada K, Hayakawa $T$, Ushio $Y$, Mitomo $M$ : Cerebral arterial dolichoectasia associated with moyamoya vessels. Surg Neurol 23: 19-24, 1985

15) Yu YL, Mosley IF, Pullicino P, Mcdonald WI: The clinical picture of ectasia of the intracerebral arteries. J Neurol Neurosurg Psychiatry 45: 29-36, 1982

Address reprint requests to: K. Matsumoto, M.D., Department of Neurosurgery, Osaka University Medical School, 1-1-50 Fukushima, Fukushima-ku, Osaka 553, Japan. 